\title{
Flicker Free VLC System with Automatic Code Resynchronization using Low Frame Rate Camera
}

\author{
Alina Elena Marcu*, Robert Alexandru Dobre ${ }^{\dagger}$, Octaviana Datcu ${ }^{\dagger}$, George Suciu ${ }^{\dagger}$, and Jeasub $\mathrm{Oh}^{\ddagger}$ \\ *Electronic Technology and Reliability Department, Politehnica University of Bucharest, Bucharest, Romania \\ Telecommunications Department, Politehnica University of Bucharest, Bucharest, Romania \\ Nano Structure Process Team 291, National NanoFab Center (NNFC), Daejeon, Republic of Korea \\ Email: alina_elena.marcu@upb.ro
}

\begin{abstract}
In the recent years, the conventional lighting systems are beginning to be replaced by LED lighting systems. In a Visible Light Communication (VLC) system, the visible light is used to provide a link between a transmitter, who is typically a $L E D$, and a receiver, which is either a photodetector or an image sensor. The VLC can be used, besides illumination, for transferring data by changing very fast the light intensity of the LED and not to be perceived by the human eye as flicker. In recent applications, high frame rate cameras have begun to be used, but their disadvantage is the high cost. This paper proposes and experimentally validates a VLC solution that uses low frame rate camera and does not require a transmitter-receiver synchronization.
\end{abstract}

Keywords - camera receiver; low frame rate camera; visible light communication;

\section{INTRODUCTION}

Over the years, the optical wireless communications (OWC) [1] began to be increasingly developed. The connectivity through the $\mathrm{OWC}$ is using as the transmission medium the visible, infrared [2] or ultraviolet light. Some examples are VLC, light fidelity (LiFi), free space optical communication (FSOC) and light detection and ranging (LiDAR). The VLC technology can be uses in several types of applications. For example, in the field of aviation, the VLC can provide media services for passengers. In the hospitals, the VLC can provide significant help in the areas where the medical equipment interferes with the radio waves [3]. In the automotive field, the VLC can be used in the Intelligent Transportation System (ITS) [4], which is composed of two systems: vehicle-to-infrastructure (V2I) and vehicle-to-vehicle (V2V) [5]. Through the V2I system, a communication between the traffic signaling system and the car can be established, thus increasing the safety of the road traffic. In the V2V system, the distance between two vehicles can be determined [6], as well as their position [7]. The ITS permanently collects information from the system about the current traffic and, in case of an emergency, adjusts the road infrastructure to that situation. Another application of VLC is in an indoor localization system, where the system uses LED beacons and a camera facing upward in order to localize the moving object in real-time [8]. The VLC system can also be implemented in smart displaying signboards, where information about the timetable of shops or

This work has been supported in part by UEFISCDI Romania and MCI through projects VLC/IR-RF, VIRTUOSE and ESTABLISH, funded in part by European Union's Horizon 2020 research and innovation program under grant agreement No. 777996 (SealedGRID project) and No. 787002 (SAFECARE project). transportation vehicles can be provided and in advertisement light panels where the information can be sent through a simple scanning of the light panels with the use of a smartphone camera [9]. In recent applications, photodetectors are replaced by image sensors [10], which have the advantages of receiving data transmitted by multiple individual LEDs [11] and the possibility to receive simultaneously data from different sources. The existing solutions are using high frame rate cameras, with over 800 frames per second [12], which are very expensive. A more affordable solution is using a camera with low frame rate, but in the current solutions, a synchronization between the image frame and the timing of the transmitted symbol is needed [13].

Besides this introduction, the paper contains four sections as follows: Section II describes the challenges in designing a VLC communication code used for a frame rate of 60 frames per second and presents the proposed code, Section III presents the experimental setup and results, and Section IV concludes the paper.

\section{The COMmunicAtion CODE}

\section{A. Design Requirements}

For a VLC system to be used by the general public, it must be designed to use affordable technology. A light sensor that many users possess is their smartphone's camera. There are two key parameters in the design of such a system: the rate at which the LEDs [14] playing the role of the transmitter are switched on or off and the frame rate of the camera used as the receiver. The following aspects must be taken into consideration in the design phase:

- A higher switching rate of the LEDs would require a high frame rate camera to correctly sample the states of the LEDs and allow a correct detection. The advantage of this case is a higher bit rate. The great disadvantage is that high frame rate cameras are very expensive.

- The switching rate of the LEDs can only be decreased to a limit to avoid the flicker effect.

- The transmitter and the receiver can be started at any moment in time and no synchronization should exist between them. The receiver should be able to automatically synchronize with the transmitter by 
receiving only the communication code and no other extra information.

It can be observed that the communication code that satisfies all the above conditions is the most important element in the whole system. The starting constraint is the highest frame rate commonly available on today's mobile terminals: 60 frames per second. Some examples of smartphones that can record video at this frame rate are: Huawei P9, Google Pixel XL, Samsung Galaxy Alpha, iPhone 7 Plus, Samsung Galaxy S7 etc.

\section{B. The Waveforms for the Proposed Communication Code}

The proposed code consists of two symbols: logical " 1 " and logical " 0 ". Each of these symbols is physically represented by an on-off switching sequence of the transmitting LEDs. Having available two symbols, a binary communication can be established. More complex codes can be imagined using the proposed symbols as a core. The sequences for the two code symbols are presented in Fig. 1 as waveforms, for better understanding. It can be observed that both symbols have a total duration of 100 milliseconds. This leads to a maximum bit rate of 10 bits per second. Since the starting condition was that the receiver is a low frame rate camera, this low bit rate was expected. The symbols are made by long and short pulses. The long pulses have a duration equal to $1 / 240$ seconds and the short ones half of that.

The transmitter and the receiver can be started at any moment in time. This determines eight possible sampling positions on the waveforms, each having 6 sampling points, illustrated in Fig. 4 to Fig. 7 on the next page. All the four possible waveform combinations were represented, and it can be observed that by following the same symbol that marks a sampling points on a waveform (i.e., x, diamond, circle etc.), four cases can be identified: "110010" or, the negated value, "001101" on a logical " 1 " waveform, no matter what is the previous symbol, and " 110011 " or " 001100 " on a logical " 0 " waveform. After one frame is captured, there are only two patterns to be detected with respect to the first captured frame, same-different-different-same-different for " 1 " symbol or
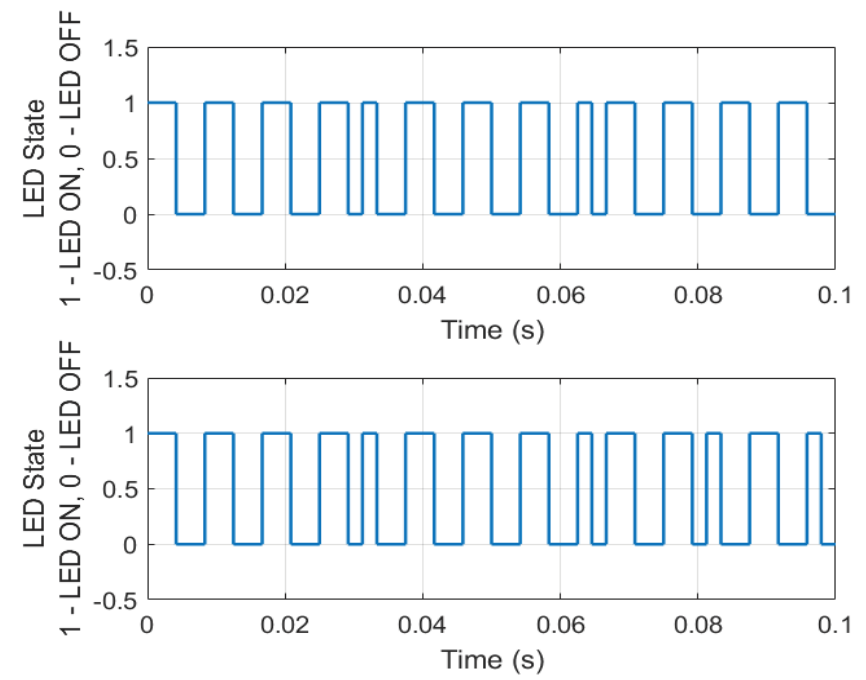

Fig. 1. The waveforms of the two symbols that form the proposed communication code: top plot for logical " 0 " and bottom for logical " 1 ". same-different-different-same-same for " 0 " symbol. In this way the system can differentiate between the two code symbols, without any synchronization available. The receiver is built as a shift register having 6 cells and its current state is polled after capturing and extracting the LED state information from each frame. If one of the previously presented patterns is identified, then the corresponding symbol is decoded, and the shift register is cleared. After a correct identification, the process starts again. If the shift register is full and no known sequence is identified, the oldest LED state in the sequence is discarded and the newest one is stored, like in a queue (FirstIn-First-Out). Problems could occur in determining the state of the LED if the sampling point is exactly on on edge of the waveform, when LED turns on or off. To counter this, two LEDs are used and the waveform that controls the second LED is obtained by delaying the main waveform by $1 / 960$ seconds (half of a short pulse). In this way, one of the two LEDs will always be sampled correctly. The detection is done identically as above, the system being run in parallel for the two LEDs.

\section{THE EXPERIMENTAL SETUP, RESUlts, AND DisCUSSION}

\section{A. The Experimental Setup and Results}

The technical setup that was used to validate the proposed communication code is presented in Fig. 2. It consists of a microcontroller development board controlling two LEDs (transmitter), and a uEye UI-1485LE-M-GL camera (receiver).

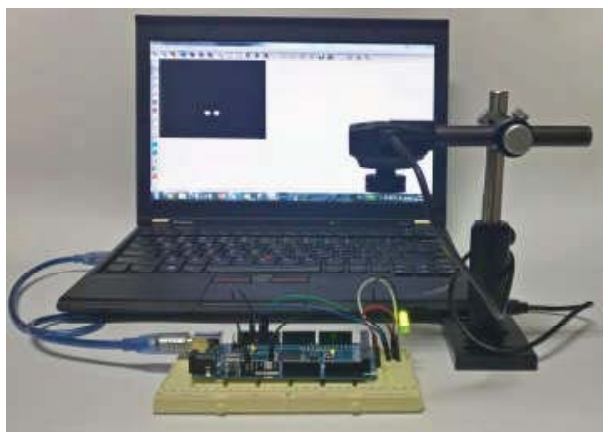

Fig. 2. The experimental setup used to validate the proposed system.

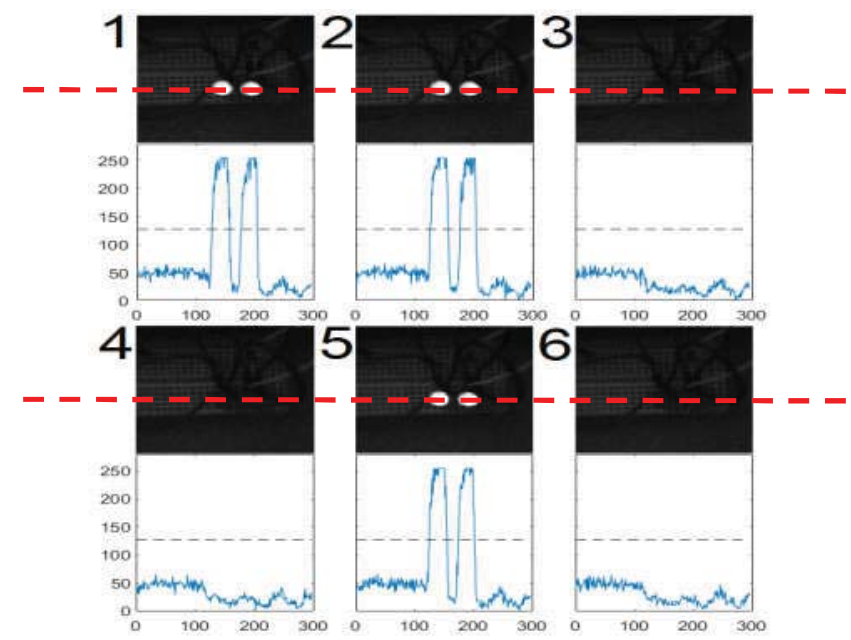

Fig. 3. The captured frames and the brightness on the probe line (red thick dashed line) in each frame (brightness of each pixel: 0 - black, 255 - white). The brightness threshold is illustrated with black thin dashed lines. 


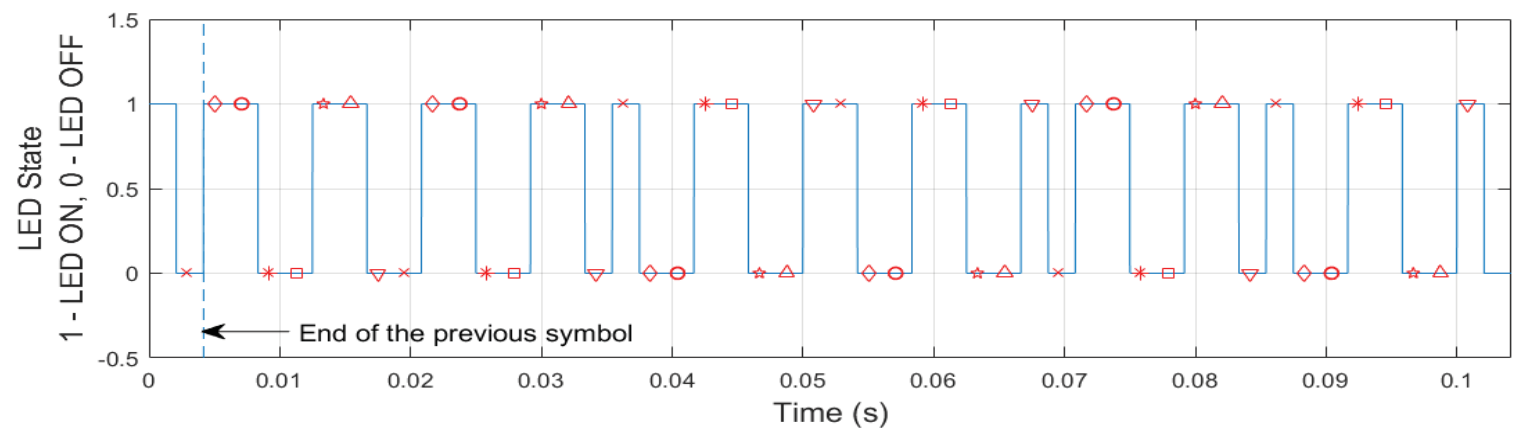

Fig. 4. Possible sampling points on the waveform of the logical "1" symbol preceded by a logical " 1 " symbol.

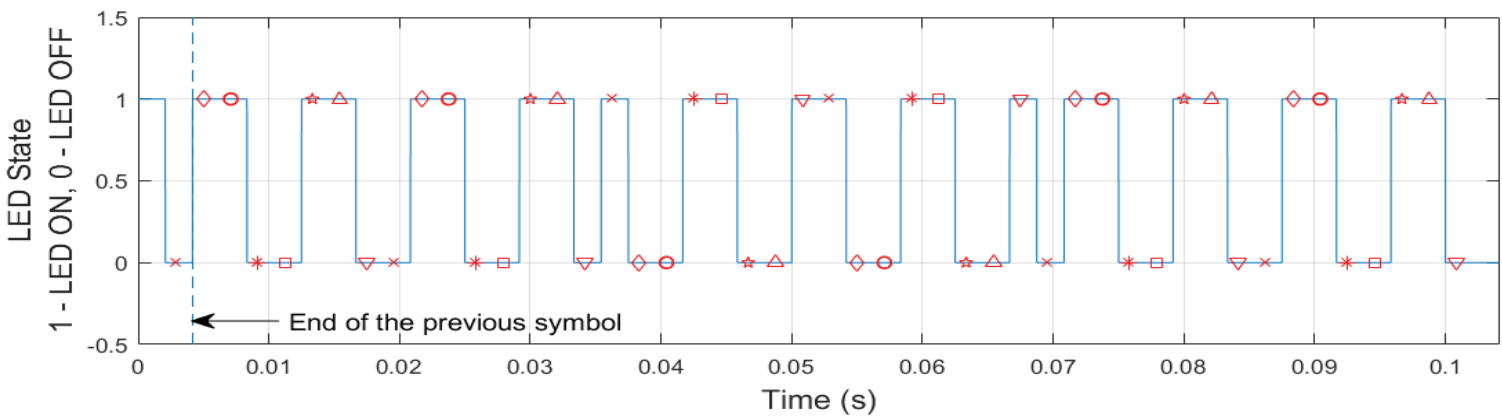

Fig. 5. Possible sampling points on the waveform of the logical " 0 " symbol preceded by a logical " 1 " symbol.

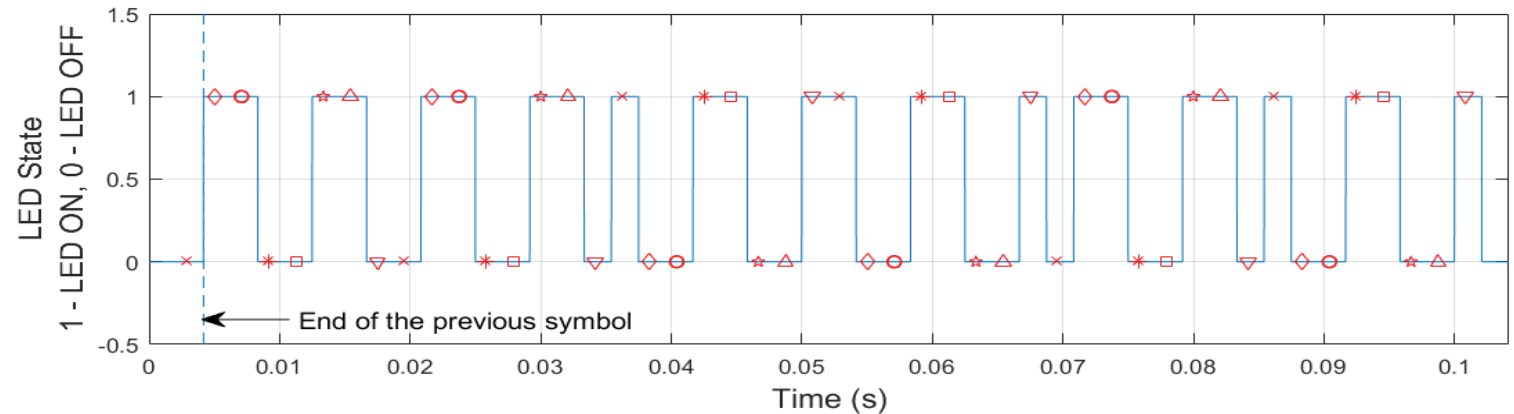

Fig. 6. Possible sampling points on the waveform of the logical "1" symbol preceded by a logical " 0 " symbol.

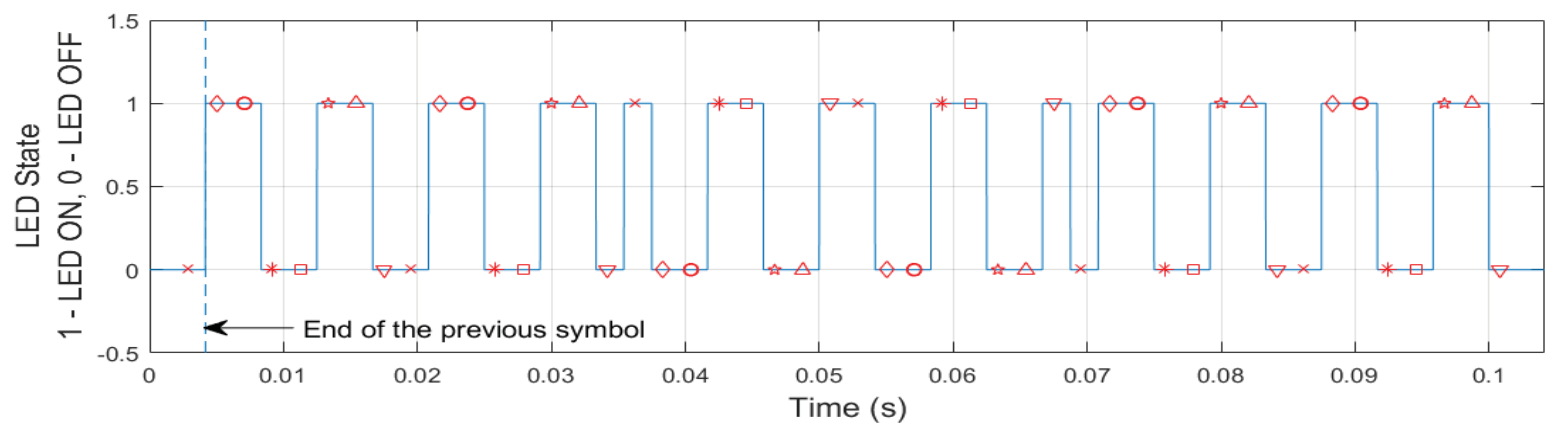

Fig. 7. Possible sampling points on the waveform of the logical " 0 " symbol preceded by a logical " 0 " symbol.

Fig. 3 illustrates the captured frames and the brightness along the probe line containing the LEDs.

The camera was set to capture 60 frames per second. The resolution of the captured frames was 296 by 296 pixels. After the video sequence has been recorded, it was analyzed frame by frame using Matlab. The two LEDs were identified in the frames and their brightness was extracted, compared with a threshold (illustrated with thin black dashed lines in Fig. 3), to get the LED state, then the state was stored in a shift register, as presented above. The threshold is computed as the mean between the minimum and the maximum brightness recorded on the probe line in 12 consecutive frames during system 
initialization. In the presented case, the identified sequence is "110010" for both LEDs, so the decoded symbol is a logical "1". The distance between the LEDs and the camera in this code validation experiment was around $10 \mathrm{~cm}$.

Due to the low bit rate, the system can be used in situations where an index, represented on maximum 10 bits is transmitted periodically. For example, in an art gallery where many works are presented, the lamps that light the artworks can use this system to send the index of each exhibited piece. This can be scanned using a mobile application dedicated to the respective gallery that can read the index as presented in this paper and show the user all the details about the work, details already stored in the app or on a web page. This is similar to the QR or barcode identification but does not need any additional materials to be placed around the artworks.

\section{B. Discussion About How the System Could Work in Real- World Conditions}

The results presented above were obtained in laboratory conditions, but the situation does not differ very much from a regular real-world functioning. The proposed code works only if the receiver captures video at 60 frames per second. If this system would be produced, it will be compatible only with smartphones or other video recording devices that capture video with the necessary frame rate. The developer must check the specifications of the devices on which the system is to be implemented and make sure that they can capture video with precisely 60 frames per second.

There is one necessary lighting condition: the light that is emitted by the transmitter LEDs should be much more intense than the ambient lighting. The system can automatically calibrate itself to the conditions thanks to the averaging done in the first 12 consecutive frames, as described above. The use of error correction codes is strongly recommended because if only one sample is missed the whole symbol is lost, but the code can resynchronize itself with the next symbol.

\section{CONCLUSION}

The paper proposes an innovative camera-based VLC system that uses LEDs as the transmitter and a camera with a low frame rate as the receiver. Furthermore, the unique propriety of the proposed code is that the transmitter and the receiver can be started at any moment in time, a synchronization between them being unnecessary. To avoid detection errors caused by capturing the frames exactly when a LED turns on or off, the code controls two LEDs: one of them is controlled directly and the other by a delayed replica of the transmitted code. In this way it is certain that at least one LED will have a clear state (fully on or fully off) when the frames are captured.

The system proposed in the paper was validated using a microcontroller development board controlling two LEDs, that play the role of the transmitter, and a uEye UI-1485LE-M-GL camera, as the receiver. The camera was set to capture 60 frames per second. The luminance of the LEDs was extracted from each video frame and comparing the resulted value with a threshold, the state of each LED was determined. By monitoring the state of the LEDs from frame to frame, the code symbols can be identified. The experimental results were presented.

The natural extension of this work in the future is represented by the evaluation of the proposed system's performances at various distances between the transmitter and the receiver and assessing the benefits of using error correction codes. After determining the maximum working distance, a multiple transmitter scenario will be studied to determine the possibility of increasing the bit rate.

\section{REFERENCES}

[1] M. Z. Chowdhury, M. T. Hossan, A. Islam, and Y. M. Jang, “A Comparative Survey of Optical Wireless Technologies: Architectures and Applications," in IEEE Access, vol. 6, 2018, pp. 9819-9840.

[2] A. Drumea, M. Blejan and C. Ionescu, "Differential inductive displacement sensor with integrated electronics and infrared communication capabilities," in Proc. SPIE 8411, Advanced Topics in Optoelectronics, Microelectronics, and Nanotechnologies VI, vol. 8411, 2012, pp. 841116.

[3] J. J. George, M. H. Mustafa, N. M. Osman, N. H. Ahmed and D. M. Hamed, "A Survey on Visible Light Communication," in International Journal of Engineering and Computer Science, vol. 3, no. 2, 2014, pp. 3805-3808.

[4] A. M. Căilean and M. Dimian, "Current Challenges for Visible Light Communications Usage in Vehicle Applications: A Survey," in IEEE Communications Surveys \& Tutorials, vol. 19, no. 4, IEEE, 2017, pp. 2681-2703.

[5] I. Takai, T. Harada, M. Andoh, K. Yasutomi, K. Kagawa, and S. Kawahito, "Optical Vehicle-to-Vehicle Communication System Using LED Transmitter and Camera Receiver," in IEEE Photonics Journal, vol. 6, no. 5, Oct. 2014, pp. 1-14.

[6] V. T. B. Tram and M. Yoo, "Vehicle-to-Vehicle Distance Estimation Using a Low-Resolution Camera Based on Visible Light Communications," in IEEE Access, vol. 6, 2018, pp. 4521-4527.

[7] T. Do and M. Yoo, "Visible Light Communication-Based Vehicle-toVehicle Tracking Using CMOS Camera,” in IEEE Access, vol. 7, 2019, pp. 7218-7227.

[8] M. Rátosi and G. Simon, "Real-Time Localization and Tracking Using Visible Light Communication," in 2018 International Conference on Indoor Positioning and Indoor Navigation (IPIN), 2018, pp. 1-8.

[9] C. Chow, R. Shiu, Y. Liu, C. Yeh, X. Liao, K. Lin, Y. Wang, and Y. Chen, "Secure Mobile-Phone Based Visible Light Communications With Different Noise-Ratio Light-Panel," in IEEE Photonics Journal, vol. 10, no. 2, April 2018, pp. 1-6.

[10] T. Yamazato, I. Takai, H. Okada, T. Fujii, T. Yendo, S. Arai, M. Andoh, T. Harada, K. Yasutomi, K. Kagawa, and S. Kawahito, "Image-sensorbased visible light communication for automotive applications," in IEEE Communications Magazine, vol. 52, no. 7, 2014, pp. 88-97.

[11] C. Premachandra, T. Yendo, M. P. Tehrani, T. Yamazato, H. Okada, T. Fujii, and M. Tanimoto, "Outdoor Road-to-Vehicle Visible Light Communication Using On-Vehicle High-Speed Camera," in International Journal of Intelligent Transportation Systems Research, vol. 13, no. 1, 2015, pp. 28-36.

[12] S. Iwasaki, C. Premachandra, T. Endo, T.i Fujii, M. Tanimoto, and Y. Kimura, "Visible light road-to-vehicle communication using high-speed camera," in 2008 IEEE Intelligent Vehicles Symposium, 2008, pp. 1318.

[13] T. Kondo, R. Kitaoka, and W. Chujo, "Multiple-access capability of LED visible light communication with low-frame-rate CMOS camera for control and data transmission of mobile objects," in 2015 IEEE/SICE International Symposium on System Integration (SII), 2015, pp. 678683.

[14] C. Marghescu and A. Drumea, "Embedded systems for controlling LED matrix displays," in Proc. SPIE 10010, Advanced Topics in Optoelectronics, Microelectronics, and Nanotechnologies VIII, vol. 10010, 2016, pp. 100101E. 